\title{
Research and Development of Miniaturized Electrets
}

\author{
J. A. Voorthuyzen, W. Olthuis, \\ P. Bergueld \\ University of 'Twente, Enschede, \\ and A. J. Sprenkels, \\ Twente Technology Transfer, Enschede, \\ The Netherlands
}

\begin{abstract}
This paper describes the realization process of small-size electrets by using techniques generally applied in the fabrication of integrated circuits and microsensors. In the first part of the paper, attention is paid to the different electret decay mechanisms and their relative contribution to the overall stability of miniaturized electrets. Then, a process is described by which polymer electrets such as Teflon-FEP and PTFE can be deposited and shaped in a predefined pattern on a silicon wafer. In the third part, results on the application of new materials, especially silicon dioxide $\left(\mathrm{SiO}_{2}\right)$, for use in electret applications, are presented. It appears that after an appropriate treatment of the oxide surface, its charge-stability is at least equal to that of polymer electrets such as Teflon-FEP and PTFE.
\end{abstract}

\section{INTRODUCTION}

\begin{abstract}
A Bout twenty years ago the interest in small sensors $A$ and actuators that perform a physical to electrical and electrical to physical conversion, started to grow rapidly. Using technologies developed for the fabrication of integrated circuits, it becomes possible to realize sensors with sub-millimeter dimensions, with comparable or even better sensitivity and long-term stability than their large predecessors.
\end{abstract}

Nevertheless the research on small, integrated electret-based sensors was not started until about 1980 , when our group initiated a research program on this subject, of which detailed results have been reported elsewhere $[1-5]$. Besides the fact that in most cases electretbased sensors and silicon-based microsensors are being investigated in two different 'research worlds', the main reason for this time-delay is the fact that the realization of electrets with sub-millimeter dimension on a silicon wafer introduces specific problems.

First of all, in Section 2 we will point out that downscaling of the lateral electret dimensions as well as the electret thickness will influence the relative contribution of different decay mechanisms on the overall stability of these electrets. We will show that in electrets with small lateral dimensions the presence of a surface-conductive layer may become important or even dominant.

Other aspects of integrating polymer electrets such as Teflon ${ }^{\text {IM }}$-FEP on a silicon wafer are the deposition and adhesion on a silicon wafer and the shaping of these layers. In Section 3 the most important aspects of how 
to integrate polymer-based electrets and silicon-based sensors are discussed. A remaining drawback of these polymers, however, is the fact that the polymer deposition and patterning is only part of the total process necessary for integrated electret-silicon sensors. Silicon processing often requires high-temperature steps. The temperature at which polymer electrets decompose $\left(\sim 450^{\circ} \mathrm{C}\right)$, is in silicon sensor processing generally considered to be a low temperature. Oxidation of silicon for instance occurs at $1 \sim 1050^{\circ} \mathrm{C}$. It will therefore be clear that polymers are hardly compatible with silicon sensor processing and that its use limits the possible sensor realizations, because only specific processing sequences are allowable.

For these reasons we have investigated the application of silicon compatible inorganic insulators as electrets. We have found that $\mathrm{SiO}_{2}$ can be used as an electret if it is pretreated in an appropriate way. The main results are presented in Section 4. In Section 5 some general conclusions are given.

To determine the charge stability in different electrets, we have applied the non-destructive compensating charge measuring method, based on the vibrating reed principle, for all measurements presented in this paper [6]. We have used a commercially available electrostatic voltmeter (Monroe isoprobe 244). In this set-up the measuring probe is at a stationary position of 0.3 to 0.6 $\mathrm{mm}$. above the electret surface, and the voltage on the probe is adjusted by a feedback loop in such a way that the electric field between probe and electret vanishes. This implies that the probe voltage equals the electret voltage. We have performed room-temperature decay as well as so-called charge-thermally stimulated discharge (charge-TSD) measurements with this probe [6].

\section{A DISCUSSION ON CHARGE DECAY MECHANISMS}

T $\mathrm{N}$ order to be able to evaluate the experimental results of $\mathrm{SiO}_{2}$ as well as Teflon-FEP electrets we shortly discuss the different charge decay mechanisms, because in reducing the lateral dimensions of electret samples, other decay mechanisms may become dominant compared to those in large samples.

To describe the different mechanisms we use the following definitions. The charge in the electret generated by any well-defined charging method is called the initial electret charge. All other charge present in the electret or at its surface, which is induced by the initial electret charge, is called the compensating electret charge. The total amount of charge in the electret, including its surface charge, is the sum of initial electret charge and compensating electret charge, and will be indicated as the net electret charge.

In many applications one-side metallized electrets are used, while a second conductive electrode is placed at a small distance above the electret surface. Between this electrode and the electret surface a small air-gap is present. Often only the external electrostatic field in the air-gap generated by the electret charge is of importance. The external electric field depends on the net electret charge as well as on the position where the charge is located in the electret. Charges present in the electret near to the metallized side for instance, hardly contribute to the external electrostatic field. Therefore electrets are generally characterized by their equivalent electret voltage (which can be measured directly by the Monroe probe mentioned above) and not by their net electret charge [6]. In the following part of this paper we define electret decay as a reduction of the initial electret voltage.

Several electret decay mechanisms are known, which have been investigated extensively by a number of authors [7-9]. Three main decay mechanisms, which determine the life-time of monopolar electrets, can be distinguished [6].

\subsection{ATTRACTION OF ENVIRONMENTAL CHARGE TO THE ELECTRET SURFACE}

In the environmental air, ions or other charged particles, of opposite polarity relative to the electret charge, are always present. These charges are attracted towards the electret surface due to the external electrostatic field, generated by the net electret charge. If these charged particles are trapped at the electret surface, they compensate the initial electret charge, and thus reduce the net electret charge as well as the external electric field and the electret voltage.

Since this decay mechanism is directly related to the number of charged particles in the air adjacent to the surface, the conditions under which the electret has been stored are of great importance [10]. It should be noted that the chance per unit area that a charged particle will reach the electret surface does not depend on the size of this surface. This means that the influence of the attraction of compensating charges on the electret decay does not depend on the lateral electret dimensions.

However, the thinner the electret, the larger the amount of charge required to achieve the same electret voltage. The attraction of compensating charges from 
the environment depends on the external electric field, generated by the electret. Assuming that other conductors, which may influence this electric field, are far away from the electret surface as compared to the electret thickness, the external electric field linearly depends on the electret voltage, and not the electret charge. This means that the electret charge decay rate (the amount of charge per second that strikes the electret surface) also linearly depends on the electret voltage. Due to the fact that the total amount of charge in a thinner electret with the same electret voltage is larger, we therefore expect that in thinner electrets with the same electret voltage as their thicker counterparts, the environmental influence will be less.

\subsection{BULK CONDUCTION}

It is assumed that the initial electret charges (mostly electrons) are trapped at localized states in the electret, from which they hardly can escape at room temperature. At an elevated temperature a fraction of these charges may be detrapped. These mobile charges will be accelerated by the electric field inside the electret, generated by the net electret charge distribution, and move towards the reverse side where they may recombine with charges of opposite polarity allocated here. The following should be noted with respect to the initial electret charge detrapping mechanism. In electret material, traps with different activation energies may be present. This implies that trapped charges will be detrapped at different temperatures. In Section 4.4 it is shown that for Teflon-FEP and $\mathrm{SiO}_{2}$ electrets two types of traps may be distinguished, which release their charges at specific temperatures.

The reverse mechanism, by which charges with opposite polarity, present at the metallized reverse side of the electret, are injected into the electret due to the internal electric field, may also occur. These charges then may move to the top side of the electret in a similar way as the initial electret charge moves to the reverse side of the electret. We can hardly distinguish both mechanisms, because both will result in a reduced electret voltage.

The electret voltage decay is thus a function of the ability of charges to move through the electret, and therefore depends on the intrinsic bulk conductivity of the electret material.

An interesting feature of bulk conduction is that the associated lifetime of electrets, in which this decay mechanism is dominating, does not depend on the actual electret dimensions. This can be explained as follows.
Assume a bulk conductivity $\rho(\Omega \mathrm{cm})$ and a permittivity $\varepsilon(\mathrm{F} / \mathrm{cm})$ of an electret with area $A$ and thickness $s$, as shown in Figure 1a. The resistance $R$ from top to bottom can thus be denoted as $R=\rho s / A$ and the capacitance as $C=\varepsilon A / s$. This means that the time constant $\tau$ determining the charge decay equals $\tau=R C=\rho \varepsilon$, which quantity is independent of the lateral dimensions as well as the thickness of the electret. The time constant $\tau$ is often known as the relaxation time, and as will be clear, is a function of the specific electret material.

\subsection{LATERAL SURFACE CONDUCTION}

To obtain stable electrets it is of utmost importance that the lateral conductivity at the air-electret interface is extremely small. Reasons for a surface conductive film at that interface may be contamination or, considering the use of electrets in normal ambient conditions, the adsorption of polar gases such as $\mathrm{H}_{2} \mathrm{O}$ from the environmental air. The relative influence of a conductive surface film on an electret increases dramatically for decreasing lateral dimensions, but on the other hand decreases for thinner electrets.

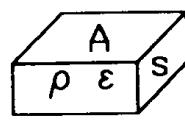

fig. a)

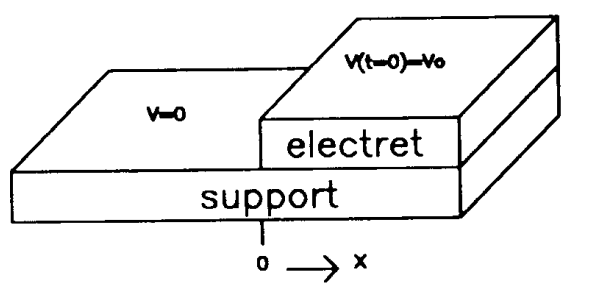

fig. b)

Figure 1.

Schematic representation of different electret geometries.

To explain this we consider the one-dimensional situation that a grounded conductive back-plate is partly covered with an electret (the region $x \geq 0$ ) and not covered in the region $x<0$, as shown in Figure $1 \mathrm{~b}$. We assume that the electret is charged initially to a voltage $V_{11}$ at $t=0$, and that the capacitance of the electret per unit area is $C$. We also assume that on top of the electret an extremely thin conductive layer with resistivity $\rho$ and thickness $d$ is present, and that the strip as drawn 
in Figure $1 \mathrm{~b}$ has a unity width. In that case the current $I$ in the $x$ direction can be written as:

$$
I=\frac{d}{\rho} E=\frac{E}{R_{l}}
$$

with $R_{l}$ the lateral surface resistance per unit area and $E$ the electric field in the $x$ direction. The electric field $E$ equals

$$
E=-\frac{d V}{d x}
$$

with $V$ the electret surface potential. Due to the fact that the electret has a capacitance $C$ per unit area, a surface potential $V$ corresponds with a surface charge $Q$

$$
Q=C V
$$

Finally the surface charge $Q$ and the lateral current $I$ are related to each other by means of the charge continuity equation

$$
\frac{d Q}{d t}=-\frac{d I}{d x}
$$

Combining these equations, we obtain the following differential equation

$$
\frac{d V}{d t}=\frac{1}{R_{l} C} \frac{d^{2} V}{d x^{2}}
$$

Accounting for the boundary conditions mentioned above, it can be shown that the solution of this equation can be written as

$$
V(x, t)=V_{0} \operatorname{erfc}\left[\left(\frac{R_{l} C x^{2}}{4 t}\right)^{0.5}\right]=V_{0} \operatorname{erfc}(\tau)
$$

with

$$
\operatorname{erfc}(\tau)=1-2 \pi^{-0.5} \int_{0}^{\tau} e^{-s^{2}} d s
$$

and $x \geq 0, t \geq 0$.

If at $t=t_{1}$ at distance $x_{1}$ the electret voltage $V\left(x_{1}, t_{1}\right)$ has reached, e.g. $90 \%$ of $V_{0}$, at distance $x_{2}$ the same value will be reached at $t=t_{2}$ with $t_{2} / t_{1}=$ $\left(x_{2} / x_{1}\right)^{2}$. This implies that if a lateral surface conduction determines the electret lifetime and the lateral dimension is reduced with a factor 10 , the lifetime at the center of the electret is reduced by a factor 100 .

Considering the expression for $V(x, t)$ we can also conclude that reducing the thickness of the electret by a factor 10, the capacitance and thus the lifetime increases by a factor 10 .

It should be noted that the presence of a surface conductive layer will also be observed if the electret is not surrounded by an electrically grounded region as drawn in Figure 1b. To explain this, we consider an electret with large lateral dimensions that is partly negatively charged and partly uncharged. At the boundary between charged and uncharged surface parts, a lateral electric field exists directed from the uncharged to the charged area. Small amount of ions will always be present in the surface conductive layer. Due to the lateral electric field the negative ions will be forced to move to the uncharged part and the positive ions to the charged part. This means that the electret voltage of the initially charged part will become less negative, while the electret voltage of the initially uncharged part will become more negative. The surface conductive layer manifests itself as a spreading of charge at the electret surface around the boundary between uncharged and charged parts. Note that the initial electret charge does not have to move, but that it is masked by a surface charge in the conductive surface layer.

\subsection{THE RELATIVE INFLUENCE OF DIFFERENT DECAY MECHANISMS}

Considering the occurrence and behavior of the different decay mechanisms mentioned in the previous Sections, the following should be noted.

In reducing the thickness of the electret the influence of the bulk conduction remains the same, while the influence of a lateral surface conduction appears to decrease. Assuming the electret voltage to be the same for thinner electrets as for their thicker counterparts, the influence of attracting compensating charge seems also to decrease. It should however be noted that we have to be careful in concluding that therefore a reduction of the electret thickness is preferable, because for thinner electrets the chance of dielectric breakdown increases, e.g. due to the presence of pinholes. For that reason we may have to permit a lower value of the electret voltage, which means a lower sensitivity of the electret-based sensor.

In reducing the lateral dimensions of the electret, the influence of bulk conduction as well as attracting compensating charge remain identical, while the influence of a lateral surface conduction increases considerably. We conclude that in reducing lateral dimensions the contribution of a lateral surface conductance may become more important and even may determine the overall long-term stability of electrets, as will be shown in a later Section on $\mathrm{SiO}_{2}$ electrets. 


\section{THE APPLICATION OF POLYMER ELECTRETS IN SILICON-BASED SENSORS}

\subsection{DEPOSITION OF POLYMER ELECTRETS}

$\mathrm{U}$

SUALLY electrets are realized by means of commercially available polymer films with a thickness of 12.5 or $25 \mu \mathrm{m}$ [6]. In many applications the thickness and the size of the electret structures are not very critical. Considering however the application of electrets in the development of silicon-based microsensors with submillimeter dimensions, the lateral dimensions of the film as well as its thickness are very important and should be controlled in a well-defined way.

The most attractive way of depositing thin polymers on a silicon wafer seems to be the on-wafer polymerization or the spin-casting and consecutive drying and sintering of a polymer suspension. Polymers fabricated in our laboratory by the last method with a thickness of 1 to $2 \mu \mathrm{m}$ appeared to have very poor chargestoring properties, probably due to an excess amount of pinholes. Therefore we have decided to use commercially available polymer films, and to deposit them by the well-known heat-sealing technique. A $10 \times 10 \mathrm{~cm}^{2}$ film is stretched several millimeters above a $5 \mathrm{~cm}$ silicon wafer, placed on a heated substrate. The air-filled gap between wafer and film is pumped down, causing the film to deflect and to be folded over the wafer. Then the substrate, wafer and film are heated to about $400^{\circ} \mathrm{C}$ so that the film is melted and sealed to the wafer.

This method has yielded a very good adhesion of polymers such as Tefion-FEP to for instance stainless steel. The adhesion of heat-sealed polymers on silicon or $\mathrm{SiO}_{2}$ in dry conditions was also sufficient, but it appeared to be reduced dramatically if the polymer-coated wafers become wetted during succeeding process steps, requiring the use of an adhesion promoter. We have found that a $1 \mu \mathrm{m}$ spin-cast polyimide suspension between the silicon wafer and the polymer electret, which is cured during the heat-sealing process, yields a strong bond [11]. The fact that in case of stainless steel supports this reduction of the bonding strength after immersion in aqueous solutions has not been observed, is due to the fact that the surface of a stainless steel support is rather rough, with numerous micropores, which are filled with the melted polymer during the heat sealing procedure. The surface of a silicon wafer is always polished and extremely smooth, because this is essential for the quality of electronic circuits made on the wafer.

\subsection{ETCHING AND PATTERNING OF POLYMER ELECTRETS}

After deposition of the polymer electret as described in Section 3.1 the whole silicon wafer is coated with a 12.5 or $25 \mu \mathrm{m}$ thick layer. For many applications this polymer should be patterned in a predefined way or etched over the whole wafer to obtain thinner electrets $[1-5]$.

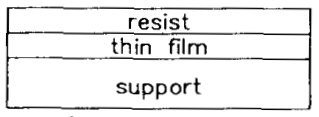

o) resist spinning

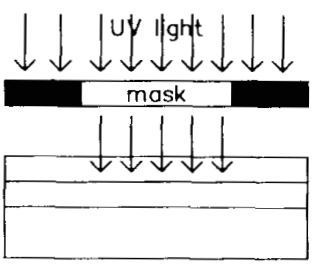

b) UV exposure

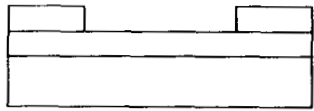

c) resist development

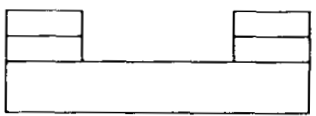

d) thin film etching

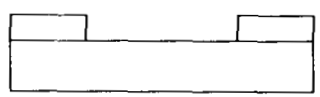

e) cleaning
Figure 2.

Consecutive steps in photolithography.

Photolithography is a very useful tool for micromachining thin films in the realization of microsensors. This technique enables us to pattern thin films in many different shapes. The process consists of several steps, as schematically drawn in Figure 2 and described below. The film, deposited on a support (usually a silicon wafer) is spin-coated with a 2 to $3 \mu \mathrm{m}$ thick layer of photoresist. The resist is pre-polymerized during $20 \mathrm{~min}$ at $90^{\circ} \mathrm{C}$. The wafer is positioned under a mask and then exposed to UV light, causing exposed parts of the resist to depolymerize (for positive resist) and non-exposed parts to remain unchanged. Then the wafer is developed and the UV-irradiated resist is dissolved. The wafer is postbaked to complete the polymerization and then placed in an etching liquid or gas, preferably specific for the material of the thin film. Finally the resist layer itself is removed and the locally etched wafer is cleaned.

The use of wet chemical etchants for photolithography is rather economical and easy, and for many materials selective etchants are known. However, for fluorocarbon polymers such etchants are not known. In the literature the use of a sodium dispersion suspended in naphthalene has been reported for the etching of Teflon, but is appears only to remove fluorine atoms from the surface, facilitating its bonding to other materials, and not to etch with a reasonable speed [12]. 


\subsection{PLASMA-ETCHING OF POLYMER ELECTRETS}

A quite different possibility for patterning of deposited polymer films is the use of a plasma in a so-called dry etching equipment. Generally a plasma is created by applying a high voltage between two electrodes in a vacuum chamber in which a certain gas is injected. For several materials, e.g. silicon and $\mathrm{SiO}_{2}$, it has been found that plasma's from $\mathrm{CCl}_{4}$ and $\mathrm{CH}_{3} \mathrm{~F}$ respectively can be used to etch these materials selectively. It has also been found that an $\mathrm{O}_{2}$ plasma can be used for the plasma etching of Teflon [13].

a)

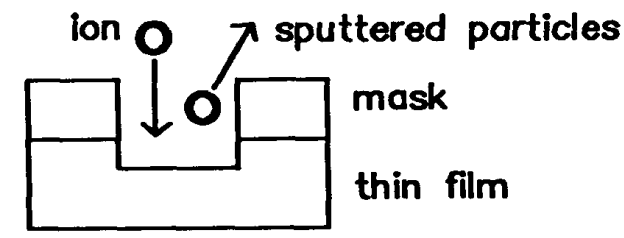

b)

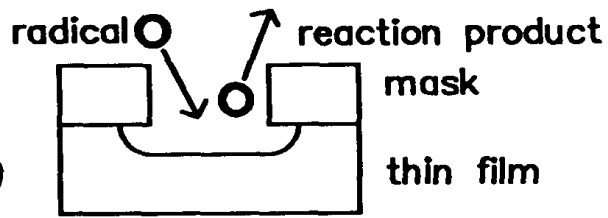

ion

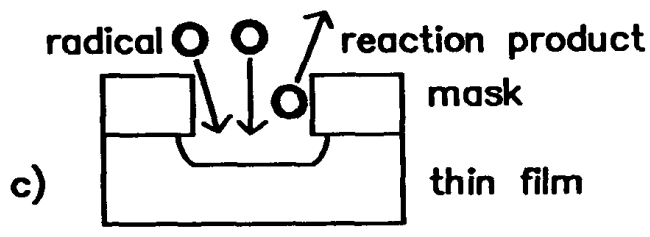

Figure 3.

Schematic etch-profiles of different etching mechanisms in a plasma-etcher, a: physical etching, b: chemical etching, c: physically enhanced chemical etching.

In a plasma, different etching mechanisms may be active, depending on the processing parameters such as power-density, gas flow and substrate temperature. The etching characteristics, e.g. selectivity (the preferential etching of one material) and anisotropy (the etching speed lateral to the substrate differs from that in the perpendicular direction) for those mechanisms are quite different, as schematically drawn in Figure 3 [14]:

\subsubsection{PHYSICAL ETCHING}

In the plasma (Figure $3 a$ ), ions are created and accelerated in the applied electric field. They strike the surface of the thin film which will be damaged and is thereby etched. Due to the acceleration of ions in one direction the etching is anisotropic, causing perpendicular profiles if a patterned masking layer is used. However, this mechanism is not very selective, because layers of different materials are etched with comparable speed.

\subsubsection{CHEMICAL ETCHING}

In the plasma (Figure $3 \mathrm{~b}$ ) also radicals of the gas are formed which may chemically react and have a preferential etching behavior for one specific material. The transport of radicals in the plasma is not determined by the applied electric field but by diffusion. This means that radicals can move in almost any direction through the reactor and thus also in a direction parallel to the film surface. This will result in so-called isotropic etching, causing considerable under-etching.

\subsubsection{PHYSICALLY ENHANCED CHEMICAL ETCHING}

A combination of both processes (Figure 3c) as described above, may of course also occur. In that case the chemical etching by radicals is enhanced or stimulated by the bombardment of the surface by accelerated ions, which cause chemical bonds to be weakened. As compared to physical etching in this situation the underetching will be larger, but on the other hand its selectivity will also be larger. The exact properties depend on the relative influence of both mechanisms on the etching characteristics, as well as on the processing parameters such as gas pressure and applied power density.

\subsection{RESULTS OF PLASMA-ETCHING OF POLYMER ELECTRETS}

We have investigated the etching characteristics of Teflon-FEP in an oxygen plasma in a barrel reactor and a plasma-etcher.

In a barrel reactor the applied electric field is parallel to the thin film surface and in a plasma-etcher the field is perpendicular to it. This means that in a barrel reactor the etching has to be of chemical origin, while in a plasma-etcher all aforementioned mechanisms may occur.

The etching of Teflon in the barrel reactor appeared to be negligible, although in the same reactor layers of 
photoresist have been etched favorably with considerable speed (about 0.1 to $0.2 \mu \mathrm{m} / \mathrm{min}$.). In the plasma etcher Teflon has been etched with a speed of about 0.15 to $0.30 \mu \mathrm{m} / \mathrm{min}$, which implies an overall etching time of about 40 to $80 \mathrm{~min}$. for $12.5 \mu \mathrm{m}$ films. The underetching appeared to be less than the initial thickness of the Teflon layer. The etching speed can be controlled by the power-density. In general it may be attractive to use the highest possible etching speed. However, we have found that in that case a residual layer of unknown composition is formed, that can not be removed. Using an $\mathrm{O}_{2}$ gas pressure of $13 \mathrm{~Pa}$ and adjusting the power in such a way that the etching speed is $0.15 \mu \mathrm{m} / \mathrm{min}$, this effect was not observed.

A masking layer is required to pattern the polymer electrets. In this case photoresist which usually has a thickness of 1 to $2 \mu \mathrm{m}$ cannot be used, because it is also etched in an $\mathrm{O}_{2}$ plasma with at least the same speed as Teflon-FEP. We have found that a $0.2 \mu \mathrm{m}$ thick layer of aluminum, deposited by evaporation and patterned by photolithography, can be used successfully. It will be clear that after the Teflon etching process, the aluminum mask has to be removed.

In order to determine which etching mechanism is responsible for the etching of polymer electrets, the following should be noted. Considering the fact that TeflonFEP cannot be etched in a barrel reactor, we can exclude that the etching is performed by radicals only. On the other hand we have seen that the etching selectivity, comparing etching rates of Teflon-FEP and aluminum, is better than 60:1, which means that we can also exclude that the etching is performed physically by ions only. This means that the etching in our plasma etcher with an $\mathrm{O}_{2}$ plasma is a physically-enhanced chemical process, which is in accordance with the observed underetching, which is less than the thickness of the Teflon-FEP layer.

In addition to the use for patterning polymer electrets, the etching process can of course also be applied for the realization of thinner electrets, by exposing the whole wafer to the plasma and controlling the etching time.

\subsection{STABILITY OF PLASMA-ETCHED TEFLON-FEP ELECTRETS}

In order to determine the influence of the plasmaetch process we have measured the long-term charge stability of different $25 \mu \mathrm{m}$ thick Tefion-FEP samples, heat-sealed on oxidized $5 \mathrm{~cm}$ silicon wafers as described in Section 3.1, following additional steps:

1. none.
2. plasma etching as mentioned in section 3.4 to a thickness of $15 \mu \mathrm{m}$.

3. as 2) with afterwards an additional 1-hour annealing at $350^{\circ} \mathrm{C}$, in order to repair possible plasma-induced damage.

4. evaporation of $0.2 \mu \mathrm{m}$ thick aluminum and subsequent removal by using a wet chemical etchant. (This layer serves as the mask material during the plasmaetching process, and can possibly contaminate the Teflon).

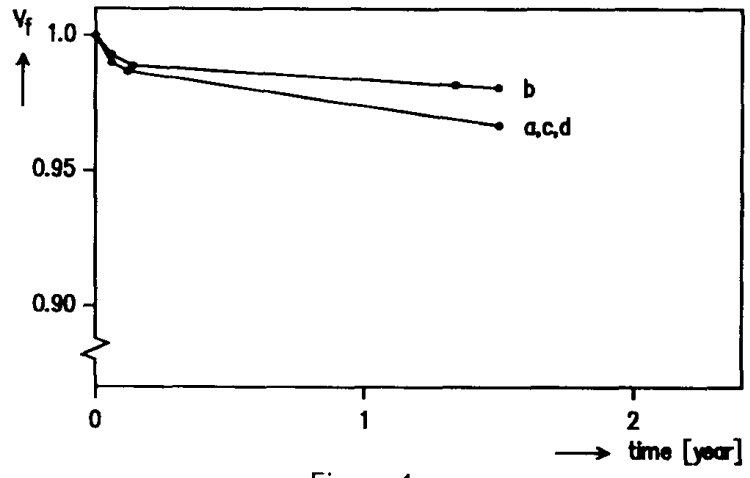

Figure 4.

Measured charge decay of SEM-charged electrets, normalized to the initial charge level. The samples have endured a different treatment (a-d) as explained in the text.

All samples have been charged using a SEM (scanning electron microscope) setup with an electret voltage from $50 \mathrm{~V}$ up to $300 \mathrm{~V}$ in $50 \mathrm{~V}$ steps, and have been measured periodically during a period of two years. All measured data, as given in Figure 4, have been normalized to the initial electret voltage. The influence of the charge level, which has been varied between $50 \mathrm{~V}$ and $300 \mathrm{~V}$, is negligible and not shown. From the results in Figure 4 we may conclude that the charge stability is only slightly affected by the aforementioned additional treatments.

\subsection{OTHER APPLICATIONS}

The plasma-etch process described in Section 3.4 can also be used for determining the average penetration depth of electron beams in flat Teflon samples. We have realized Teflon samples on a conductive support with different thicknesses by changing the etching time. The thickness has been measured by determining the weight loss after etching. In all samples the same amount of electric charge has been injected by a SEM. This implies 
that the surface potential linearly depends on the electret thickness. Plotting the surface potential, as measured by the Monroe probe, of the various electrets vs. the electret thickness, the intersection with the $x$-axis yields the average penetration depth. The results are shown in Figure 5, for samples charged with an acceleration voltage of $15 \mathrm{kV}$. We observe an average penetration depth of $2.5 \mu \mathrm{m}$, which corresponds rather well to the value reported elsewhere [6].

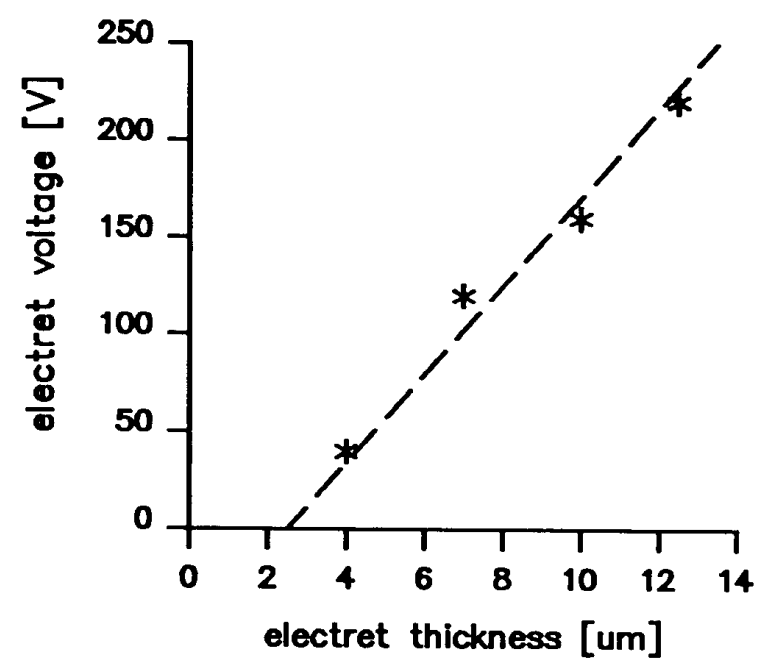

Figure 5.

Measured electret surface potential as a function of electret thickness.

Considering the results of plasma etching polymer electrets, it is concluded that it is possible to make Teflon-FEP electrets with a minimum size of about its original thickness due to the small underetching. This may be of interest in the realization of complicated electret patterns for use in electrostatic position measurement etc. In general it can be said that the number of possible polymer electret applications is no longer limited to large-size applications, but only by the imagination of the user.

\section{THE APPLICATION OF $\mathrm{SiO}_{2}$ AS ELECTRET}

A LTHOUGH many problems associated with the use of polymer electrets in silicon microstructures can be solved, as described above, the main drawback of their application is the low temperature at which they start to decompose. Therefore we decided to investigate the charge-storing properties of different inorganic materials which have a high melting or decomposition temperature and are more compatible to silicon sensor processing.
It was already known from the literature that dielectrics such as $\mathrm{Ta}_{2} \mathrm{O}_{5}, \mathrm{SiO}_{2}, \mathrm{Si}_{3} \mathrm{~N}_{4}$ and $\mathrm{Al}_{2} \mathrm{O}_{3}$ behave under certain circumstances like electrets $[15,16]$. Preliminary measurements have been carried out on these four materials in our laboratory, which have revealed a promising electret behavior for the $\mathrm{SiO}_{2}$ and $\mathrm{Al}_{2} \mathrm{O}_{3}$ samples. Based on these results and on those reported in the literature [15], as well as the fact that $\mathrm{SiO}_{2}$ is a standard material in our laboratory, we have decided to confine ourselves to further investigate the electret properties of $\mathrm{SiO}_{2}$ only. In order to compare the experimental results, those of Teflon-FEP will also be presented.

\subsection{PREPARATION OF $\mathrm{SiO}_{2}$ ELECTRETS}

All $\mathrm{SiO}_{2}$ samples have been prepared by wet thermal oxidation at $1100^{\circ} \mathrm{C}$ of previously cleaned $5 \mathrm{~cm}$ silicon wafers with a $<100>$ orientation and a 5 to $10 \Omega \mathrm{cm}$ resistivity. The average thickness of the oxide layers thus realized is about $1.1 \mu \mathrm{m}$. After oxidation, the oxide on the back of the wafer is removed by chemical etching or scratching, to allow contact to the reverse side of the wafer in the charging equipment. Most samples have been liquid-contact charged, while a few samples have been corona charged, as described elsewhere [6].

\subsection{EXPERIMENTAL RESULTS WITH VIRGIN-SiO ${ }_{2}$ ELECTRETS}

In our first experiments we have charged $5 \mathrm{~cm}$ large $\mathrm{SiO}_{2}$ samples, prepared as described in Section 4.1, by means of the liquid-contact method. These samples showed a large electret voltage decay with a time constant in the order of days. To investigate this observed voltage decay, a steep charge step has been created on an $\mathrm{SiO}_{2}$ sample, using the liquid-contact method, followed by repeated scanning the boundary with the Monroe probe. The measured decay is shown in Figure 6 . The left side has been charged up to $150 \mathrm{~V}$, while the right side remained uncharged. As is clear from this Figure, the net electret charge is spread relatively fast and evenly over the surface. This can be described only by assuming a relatively large lateral surface conduction, because a decay due to bulk conduction or the attraction of compensating charges will not be accompanied by such a charge spreading. Note that the observed behavior does not imply that the initial electret charge becomes mobile due to the presence of a surface conductive layer, but that a compensating surface charge may be generated by separation of positive and negative charges in this conductive layer. See also the discussion in Section 2.3. 


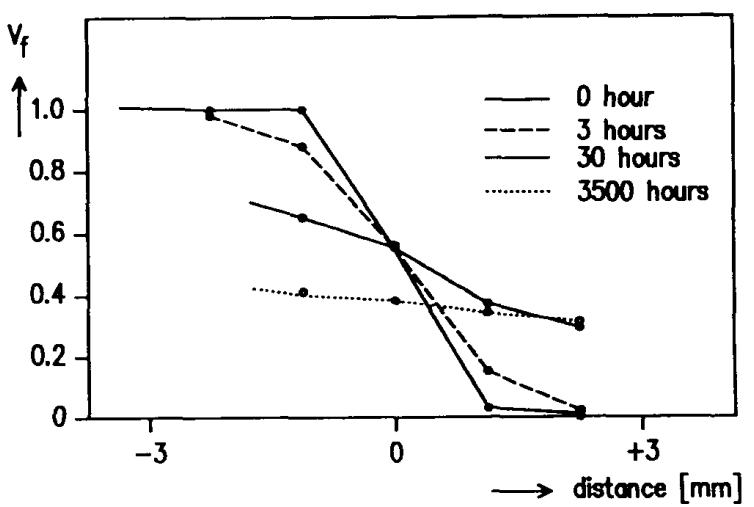

Figure 6.

Measurements showing the lateral surface conduction on a virgin $1.1 \mu \mathrm{m}$ thick $\mathrm{SiO}_{2}$ electret.

The origin of this surface conductance for $\mathrm{SiO}_{2}$ films as well as the method to reduce it will be discussed in the next Section.

\subsection{THE SURFACE CONDUCTIVITY OF $\mathrm{SiO}_{2}$}

It has been shown by several authors that the lateral surface conductivity of $\mathrm{SiO}_{2}$ is mainly determined by a chemical adsorption of polar groups (mainly water) to the $\mathrm{SiO}_{2}$ surface, forming $\mathrm{OH}$-groups [17-20]. These groups may in turn adsorp $\mathrm{H}_{2} \mathrm{O}$ groups, causing a conductive layer on top of the $\mathrm{SiO}_{2}$ surface. The physical adsorption depends on the relative humidity of the environmental air, but the chemisorption not.

The best procedure to eliminate the surface conductivity will therefore be a removal of the chemically adsorbed $\mathrm{OH}$-groups either by heat treatment at $400^{\circ} \mathrm{C}$ or by chemical surface modification $[19,20]$. We have found that heat treatment does not remove the adsorbed $\mathrm{OH}$ groups completely. A more permanent solution is obtained by a chemical surface modification, in which the polar silanol groups are substituted by apolar groups, which do not adsorb polar $\mathrm{H}_{2} \mathrm{O}$-groups. This treatment may be considered as a hydrophylic to hydrophobic conversion of the $\mathrm{SiO}_{2}$ surface and may be accomplished by treatment of the $\mathrm{SiO}_{2}$ surface with hexamethyldisilazane (HMDS) [21]. Using this treatment the oxide surface is nearly completely covered with a mono-layer of nonpolar $\mathrm{CH}_{3}$-groups, which makes any adsorption of polar $\mathrm{H}_{2} \mathrm{O}$-groups very unlikely. The result is an $\mathrm{SiO}_{2}$ surface with a very low surface conductivity [18]. Experimental results of $\mathrm{SiO}_{2}$ electrets treated in this way prior to their charging are given in the next Section.

\subsection{EXPERIMENTAL RESULTS WITH CHEMICALLY-MODIFIED $\mathrm{SiO}_{2}$ ELECTRETS}

We have measured the long-term stability of $1 \times 1$ $\mathrm{cm}^{2} \mathrm{SiO}_{2}$ samples with and without chemical surface treatment. Some characteristic results are shown in Figure 7 . The lower curves represent the charge decay of untreated $\mathrm{SiO}_{2}$ electrets, which have been charged up to $150 \mathrm{~V}$. The upper line represents the decay of an $150 \mathrm{~V}, \mathrm{HMDS}$-treated $\mathrm{SiO}_{2}$ electret. After an initial decay of about 2 to $3 \%$ during the first day, no further decay could be measured during the next 17 months. Note that this confirms the assumption that the bulk conduction of $\mathrm{SiO}_{2}$ electrets is negligible at room temperature, while the surface modification also makes the lateral conduction infinitesimally small.

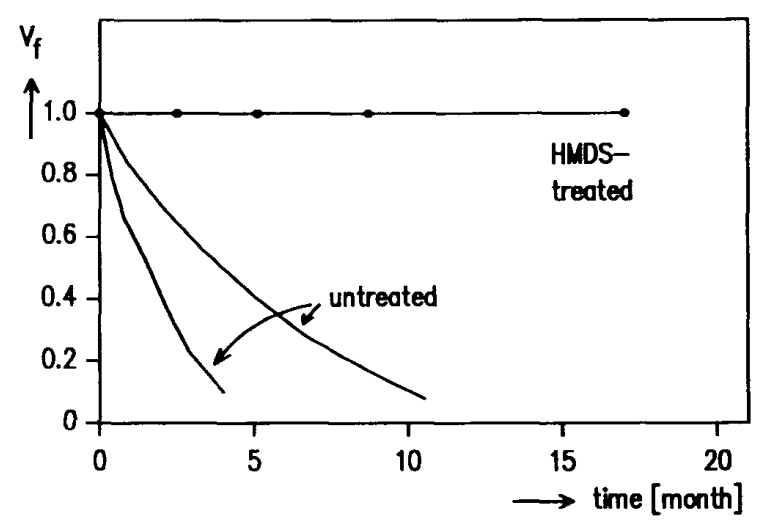

Figure 7.

Normalized charge decay at ambient laboratory conditions of some liquid-contact charged $\mathrm{SiO}_{2}$ electrets with and without an HMDS treatment.

The initial decay of 2 to $3 \%$ during the first day is probably the result of charges in shallow traps which are detrapped very easily. Experiments have shown that this decay may be artificially accelerated by curing the sample for a period of 10 to $30 \mathrm{~min}$ at $90^{\circ} \mathrm{C}$.

In order to examine the temperature dependence, the charge-TSD of HMDS-treated corona-charged $\mathrm{SiO}_{2}$ electrets has been measured, from which some results are given in Figure 8 . The temperature has been increased in $20^{\circ} \mathrm{C}$ steps of $10 \mathrm{~min}$ each, from 20 up to $260^{\circ} \mathrm{C}$ in the first run, and from 20 up to $400^{\circ} \mathrm{C}$ in the second run. After each 10 min temperature step, the samples are cooled down and measured with the Monroe probe. The results may be compared with the corresponding results in Figure 9 for a $25 \mu \mathrm{m}$ Teflon-FEP electret sample, exposed to the same procedure. Considering both 


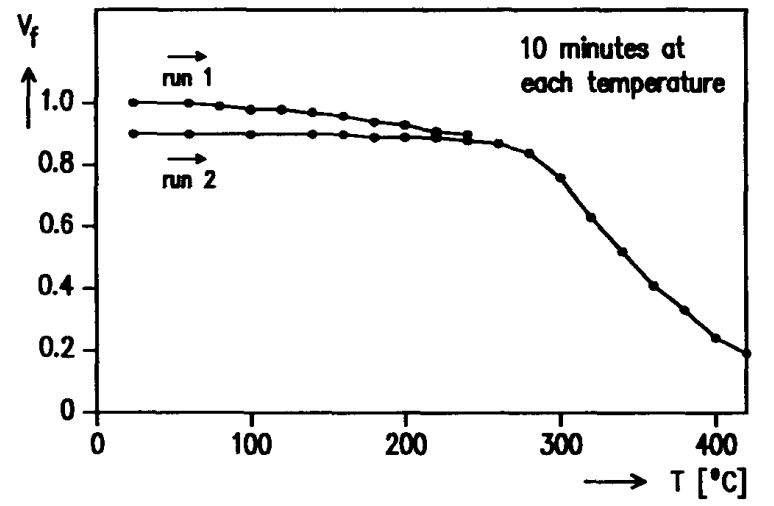

Figure 8.

Normalized charge decay of an $1.1 \mu \mathrm{m}$ thick corona-charged $\mathrm{SiO}_{2}$ electret as a function of temperature.

figures it is clear that the tendency of the charge decay of Teflon-FEP and $\mathrm{SiO}_{2}$ electrets is the same. The only difference is the fact that a higher temperature is required for an $\mathrm{SiO}_{2}$ electret to show the same decay behavior as a Teflon-FEP electret. This suggests that the trap-depth of charge in a $\mathrm{SiO}_{2}$ electret is larger than in a Teflon-FEP electret.

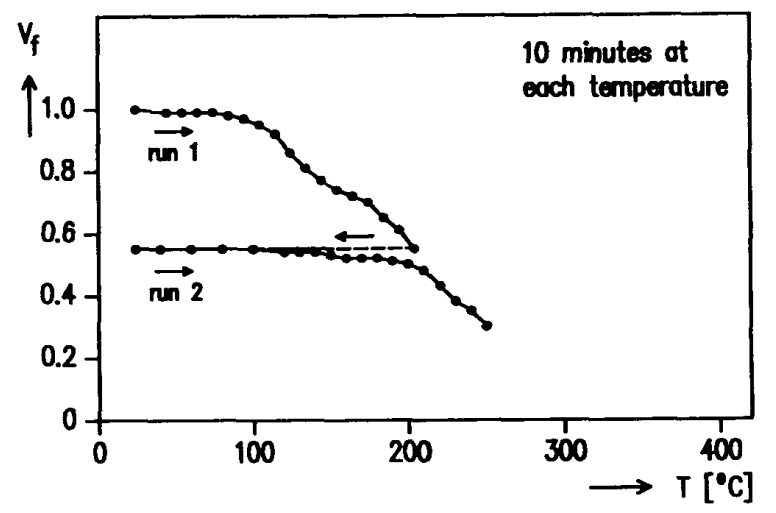

Figure 9.

Normalized charge decay of a $25 \mu \mathrm{m}$ thick corona-charged Teflon-FEP electret as a function of temperature.

This is even more clear from Figure 10, where the electret charge decay (trace 1) of an HMDS-treated liquidcontact charged $\mathrm{SiO}_{2}$ electret is shown at a constant temperature of $200^{\circ} \mathrm{C}$. During the first hour the charge decay is relatively rapid, while thereafter the decay is more or less stabilized. First the traps with a relatively low activation energy release their charges. After one hour most of these traps are empty and a second kind of traps determines the charge decay. If this sample is charged again to $150 \mathrm{~V}$, and heated in the same way, trace 2 is found. This indicates the same phenomenon as can be observed for Teflon-FEP electrets, as presented in Figure 11: a charged electret which is partly decayed by a heat treatment and subsequently charged to its initial level has become more stable, a procedure that is widely used in practice.

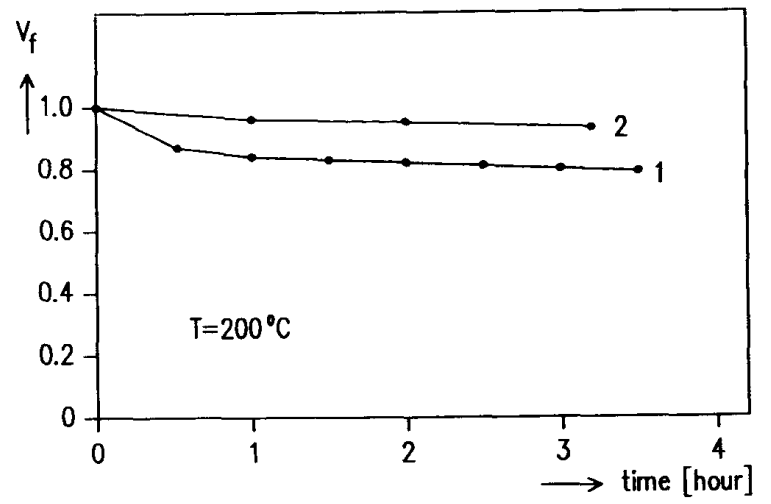

Figure 10.

Normalized isothermal charge decay of an $1.1 \mu \mathrm{m}$ thick $\mathrm{SiO}_{2}$ electret at $200^{\circ} \mathrm{C}$ (trace 1 ). If this electret sample is subsequently charged to its initial charge level, it has become better resistant to temperature (trace 2 ).

Comparing Figures 10 and 11, we may conclude that the charge decay mechanisms of Teflon and chemically treated $\mathrm{SiO}_{2}$ electrets are comparable, although occurring at different temperatures. It appears that a $1.1 \mu \mathrm{m}$ thick $\mathrm{SiO}_{2}$ electret is even more resistant to high temperatures. Assuming a comparable temperature dependence of the charge stability for both materials we can conclude that the room-temperature charge stability of chemically modified $\mathrm{SiO}_{2}$ electrets is better than of Teflon-FEP.

Finally some experiments have been carried out in order to examine the influence of the charge level on the charge stability. In Figure 12 the normalized electret decay of a number of HMDS-treated liquid-contact charged $\mathrm{SiO}_{2}$ electrets is shown for a period of 15 months. The initial charge level of the different samples ranges from 175 to $663 \mathrm{~V}$. All samples have been placed in small boxes, which has a positive influence on the electret lifetime [10]. The boxes have been stored in our laboratory, at 18 to $25^{\circ} \mathrm{C}$ in 40 to $70 \% \mathrm{RH}$. We note that is has been possible to charge $\mathrm{SiO}_{2}$ up to $663 \mathrm{~V}$, resulting however 


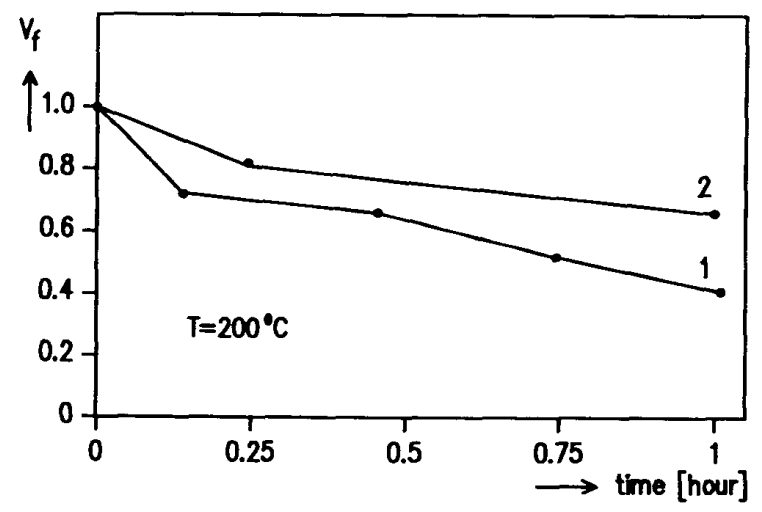

Figure 11.

Normalized isothermal charge decay of a $25 \mu \mathrm{m}$ thick Teflon-FEP electret at $200^{\circ} \mathrm{C}$ (trace 1 ). If this electret sample is subsequently charged to its initial charge level, it has become better resistant to temperature (trace 2 ).

in a slightly reduced charge stability. In our case the electric field amounts to $6 \mathrm{MV} / \mathrm{cm}$, which is very close to the theoretical maximum field strength of $7 \mathrm{MV} / \mathrm{cm}$ found by others [22]. A lower limit for the electret lifetime of $20 \mathrm{yr}$ may be estimated for the $663 \mathrm{~V}$ electret. In addition it appears from Figure 12 that the charge decay shows a weak dependence on the charge level and thus on the electric field in the oxide.

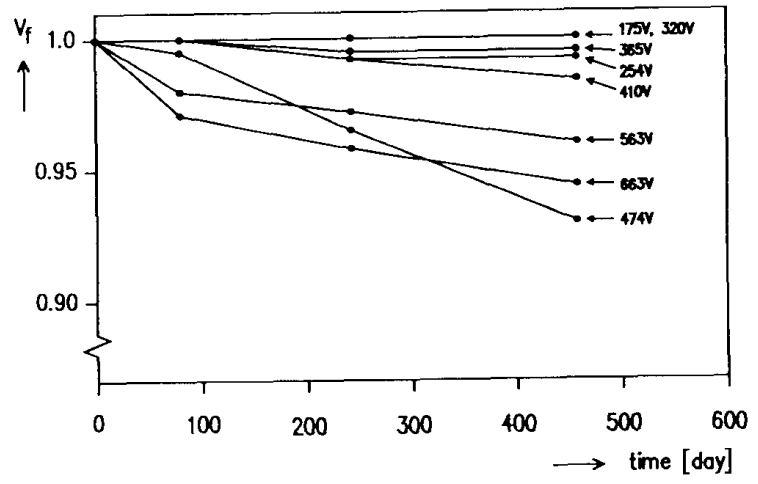

Figure 12.

Normalized charge decay of some liquid-contact charged $1.1 \mu \mathrm{m}$ thick $\mathrm{SiO}_{2}$ electrets for different charge levels.

It is noteworthy that in previous literature on $\mathrm{SiO}_{2}$ electrets the possible charge decay due to a surface conductance of this material has not been reported or observed, even not for untreated $\mathrm{SiO}_{2}$ electrets. This might be due to storing conditions that differ from those used in our laboratory, or due to the use of $\mathrm{SiO}_{2}$ electrets with much larger lateral dimensions than the usual size of $1 \times 1 \mathrm{~cm}^{2}$ we have adopted [15].

From the measured decay of HMDS-treated $\mathrm{SiO}_{2}$ samples (Figure 7) an upper limit for the bulk conductivity of $\mathrm{SiO}_{2}$ at room temperature can be calculated according to the method originally proposed by Sessler [23]. If we assume that the charge decay is less than $\frac{1}{2} \mathrm{~V}$ during 17 months (Figure 7), and only due to ohmic conduction, an upper limit for the conductivity of $\mathrm{SiO}_{2}$ is $g=2.7 \times 10^{-} 23 \Omega^{-1} \mathrm{~cm}^{-1}$, which is considerably smaller than the maximum value of about $10^{-} 18 \Omega^{-1} \mathrm{~cm}^{-1}$ reported in the literature. This large difference may be due to the fact that the last value is determined by depositing a conductive electrode on top of the oxide and measuring the conductance between this electrode and the silicon support. Obviously the presence of this electrode disturbs the measurements dramatically, or influences the $\mathrm{SiO}_{2}$ bulk properties due to migration of metal ions.

\section{CONCLUSION}

T $\mathrm{HE}$ different discharge mechanisms determining or 1 restricting the lifetime of electrets have been discussed in relation to down-scaling of the electret dimensions. The most important conclusion is the fact that for reduced lateral electret dimensions the charge decay may be dominated by an electret surface conductance.

We have shown that a plasma-etching process can be used satisfactorily for the patterning of polymers and that the etching itself is mainly determined by physically enhanced chemical etching. The plasma etching process has appeared to have a negligible influence on the charge stability of Teflon-FEP electrets.

It has been demonstrated that an untreated $\mathrm{SiO}_{2}$ layer is a poor electret mainly due to its large lateral surface conduction. However we have shown that an $1.1 \mu \mathrm{m}$ thick $\mathrm{SiO}_{2}$ layer may be used as an excellent electret if the surface conductivity has been reduced by an appropriate chemical surface modification. This surface modification is adequately accomplished by a hydrophylic to hydrophobic conversion using HMDS.

It has appeared that the charge stability of HMDStreated $1.1 \mu \mathrm{m}$ thick $\mathrm{SiO}_{2}$ electrets is better than that of 12.5 and $25 \mu \mathrm{m}$ thick Teflon-FEP electrets, storing both under normal ambient conditions of temperature and relative humidity. We have not measured any charge decay at room temperature for a period of 17 months of 
HMDS-treated $\mathrm{SiO}_{2}$ electrets charged up to $145 \mathrm{~V}$. This results in a lifetime of more than $400 \mathrm{yr}$.

It is adequate to age $\mathrm{SiO}_{2}$ electrets by two or three charge-discharge cycles prior to their use. By this artificial aging the traps which cause an initial charge decay are released. This was already known for Teflon electrets, but has appeared to be valid for $\mathrm{SiO}_{2}$ electrets as well. It may be concluded that the charge decay mechanisms of Teflon and $\mathrm{SiO}_{2}$ electrets are very much alike, occurring however at different temperatures. Up to $663 \mathrm{~V}$ charged $\mathrm{SiO}_{2}$ electrets have revealed a lower limit for their lifetime of more than $20 \mathrm{yr}$.

\section{REFERENCES}

[1] J. A. Voorthuyzen and P. Bergueld, "Theoretical considerations in the design of integrated semiconductor sensors applying electrets", IEEE Trans. Electron Dev., Vol. 32, no. 7, pp. 1185-1190, 1985.

[2] A. J. Sprenkels, J'. A. Voorthuyzen and P. Bergveld, "A theoretical analysis of the electret air-gap field effect structure for sensor application", Sensors and Actuators, Vol. 9, pp. 59-72, 1986.

[3] J. A. Voorthuyzen, The PRESSFET, an integrated electret-MOSFET structure for application as a catheter-tip blood pressure sensor, Thesis, Twente University, 1986.

[4] A. J. Sprenkels, A silicon subminiature electret microphone, Thesis, Twente University, 1988.

[5] J. A. Voorthuyzen and P. Bergueld, "The PRESSFET: an integrated electret-MOSFET based pressure sensor", Sensors and Actuators, Vol. 14, pp. 349-360, 1988.

[6] G. M. Sessler, "Topics in applied physics", Vol. 33, Electrets, Springer-Verlag, Berlin, 1980.

[7] P. W. Chudleigh, R. E. Collins and G. D. Hancock, "Stability of liquid charged electrets", Appl. Phys. Lett., Vol. 23, no. 5, pp. 211-212, Sept. 1973.

[8] G. M. Sessler and J. E. West, "Charge Properties of Electron-Beam Irradiated Dielectrics", From: Electrets, charge storage and transports in dielectrics, pp. 292-299, 1973

[9] J. Roos, "Electrets, Semipermanently Charged Capacitors", J. Appl. Phys., Vol. 40, no. 8, pp. 31353139, July 1969.

[10] E. W. Anderson, L. L. Blyler, Jr. G. E. Johnson and G. L. Link, "Electret Stability in the Presence of lons", in M. M. Perlman. ed. Electrets, charge storage and transport in dielectrics, pp. 424-435, 1973.
[11] A. M. Wilson, "Polyimide insulators for multilevel interconnections", Thin Solid Films, Vol. 83, pp. 145-163, 1981.

[12] S. J. Updike, M. C. Shults and M. Busby, "Continuous glucose monitor based on an immobilized enzyme electrode detector", J. Lab. Clin. Med., Vol. 93, no. 4, pp. 518-527, 1979.

[13] L. A. Perderson, "Structural composition of polymers relative to their plasma etch characteristics", J. Electrochem. Soc., Vol. 129, no. 1, pp. 205-208, Jan. 1982.

[14] S. J. Fonash, "Advance in dry etching processes - a review", Solid-State Technol., Vol,. 28, pp. 150-158, Jan. 1985.

[15] D. Hohm and R. Gerhard-Mulhaupt, "Silicon-dioxide electret transducer", J. Acoust. Soc. Am., Vol. 75, no. 4, pp. 1297-1298, April 1984.

[16] A. E. Guile, "Stored charges in relatively thin oxide films", J. Phys. D: Appl. Phys., Vol. 5, pp. 11531156, 1972.

[17] Y. Awakuni and J. H. Calderwood, "Water vapour adsorption and surface conductivity in solids", J. Phys. D: Appl. Phys., Vol. 5, pp. 1038-1045, 1972.

[18] J. A. Voorthuyzen, K. Keskin and P. Bergveld, "Investigations on the surface conductivity of silicon dioxide and methods to reduce it", Surface Science, Vol. 187, pp. 201-211, 1987.

[19] G. J. Young, "Interaction of water vapor with silica surfaces", J. Colloid Science, Vol. 13, pp. 67-85, 1958.

[20] A. van den Berg, P. Bergveld, D. N. Reinhoudt and E. J. R. Sudhölter, "Sensitivity control of ISFETs by chemical surface modification", Sensors and Actuators, Vol. 8, pp. 129-148, 1985.

[21] H. Yanazawa, H. Utsugi, N. Hashimoto and M. Ashikawa, "Hydrophobic Conversion of the Chemical-Vapor-Deposited $\mathrm{SiO}_{2}$ Surface", Proc. 6 th Intern. Vacuum Congr. 1974 Japan J. Appl. Phys. Suppl. 2 Pt. 1, pp. 753-756, 1974.

[22] R. Williams and H. Woods, "High electric fields in silicon dioxide produced by corona charging", J. Appl. Phys., Vol. 44, no. 3, pp. 1026-1028, March 1973.

[23] G. M. Sessler and J. E. West, "Foil-Electret Microphones", J. Acoust. Soc. Am., Vol. 40, no. 6, pp. 1433-1440, 1966.

Manuscript was received on $17 \mathrm{Feb} 1989$, in revised form 6 Mar 1989. 